\title{
Performance versus Risk Factor-Based Approaches to Coronary Artery Disease Screening in Waitlisted Kidney Transplant Candidates
}

\author{
Xingxing S. Cheng a Daniel J. Watford ${ }^{\mathrm{a}}$ Hiroyuki Arashi ${ }^{\mathrm{b}, \mathrm{c}}$ \\ Margaret R. Stedman ${ }^{a}$ Glenn M. Chertow ${ }^{a}$ Jane C. Tan ${ }^{a}$ William F. Fearon ${ }^{b}$ \\ aDivision of Nephrology, Department of Medicine, Stanford University School of Medicine, Stanford, CA, \\ USA; ${ }^{b}$ Division of Cardiovascular Medicine, Department of Medicine, Stanford University School of Medicine, \\ Stanford, CA, USA; 'Department of Cardiology, Tokyo Women's Medical University, Tokyo, Japan
}

\section{Keywords}

Coronary artery disease $\cdot$ Screening $\cdot$ Kidney transplantation · Six-minute walk test

\begin{abstract}
Introduction: Current screening algorithms for coronary artery disease (CAD) before kidney transplantation result in many tests but few interventions. Objective: The aim of this study was to study the utility of 6-minute walk test (6MWT), an office-based test of cardiorespiratory fitness, for risk stratification in this setting. Methods: We enrolled 360 patients who are near the top of the kidney transplant waitlist at our institution. All patients underwent CAD evaluation irrespective of $6 \mathrm{MWT}$ results. We examined the association between 6MWT and time to CAD-related events (defined as cardiac death, revascularization, nonfatal myocardial infarction, and removal from the waitlist for CAD), treating noncardiac death and waitlist removal for non-CAD reasons as competing events. Results: The 6MWT-based approach designated approximately $45 \%$ of patients as "low risk," whereas a risk factor- or symptom-based approach designated 14 and $81 \%$ of patients as "low risk," respectively. The 6MWT-based approach was not significantly associated with CAD-related events within 1 year (subproportional hazard ratio [sHR] 1.00
\end{abstract}

[0.90-1.11] per $50 \mathrm{~m}$ ) but was significantly associated with competing events (sHR 0.70 [0.66-0.75] per $50 \mathrm{~m}$ ). In a companion analysis, removing waitlist status from consideration, 6MWT result was associated with the development of CADrelated events (sHR 0.92 [0.84-1.00] per 50 m). Conclusions: The 6MWT designates fewer patients as high risk and in need of further testing (compared to risk factor-based approaches), but its utility as a pure CAD risk stratification tool is modulated by the background waitlist removal rate. CAD screening before kidney transplant should be tailored according to a patient's actual chance of receiving a transplant.

(c) 2021 The Author(s)

Published by S. Karger AG, Basel

\section{Introduction}

Kidney transplant, the preferred treatment for advanced chronic kidney disease (CKD) and end-stage kidney disease (ESKD), is an elective surgical procedure. Screening for coronary artery disease (CAD) prior to transplant has been and remains a key part of the pretransplant evaluation [1]. The optimal, evidence-based approach to screening asymptomatic patients, however, is far from clear. karger@karger.com www.karger.com/crm

Karger $\frac{1}{\%}$
(C) 2021 The Author(s)

Published by S. Karger AG, Basel

This is an Open Access article licensed under the Creative Commons Attribution-NonCommercial-4.0 International License (CC BY-NC) (http://www.karger.com/Services/OpenAccessLicense), applicable to the online version of the article only. Usage and distribution for commercial purposes requires written permission.
Correspondence to:

Xingxing S Cheng, xscheng@stanford.edu 
Recent advances have questioned the utility of CAD screening, especially as revascularization of stable, asymptomatic $\mathrm{CAD}$ lesions has not been shown to improve outcomes in multiple patient populations [2, 3]. However, in practice, pretransplant screening remains ubiquitous [4]. A rational first step may be thus to reduce the number of patients screened, starting with risk stratification that appropriately focuses testing on a subset of highrisk patients while shielding low-risk patients from unnecessary testing. Current guidelines $[5,6]$ risk-stratify patients based on traditional CAD risk factors. However, these risk factors "rule in" most kidney transplant candidates for testing. By definition, 100\% of kidney transplant candidates have advanced CKD, a CAD risk factor. Furthermore, most patients with CKD have additional CAD risk factors. In a multicenter cohort of kidney transplant recipients, diabetes mellitus, dyslipidemia, smoking, and CAD or CAD-equivalents were present at $26,36,35$, and $14 \%$, respectively [7]. In another cohort, $92 \%$ of transplant candidates qualified for cardiac testing under risk factor-based guidelines [8]. Despite this high "rule in" rate, the intervention rate was only $9.5 \%$ in a study of Medicare beneficiaries with ESKD [9].

Risk stratification might otherwise be accomplished by physical performance testing. Ability to achieve $\geq 4$ metabolic equivalent of tasks (METs) is an early branch point in the screening algorithm for elective, non-cardiac surgeries in the 2014 ACC/AHA guideline [10]. Two studies to date have considered physical performance in risk stratification prior to kidney transplantation. In one, self-reported physical performance was not sensitive for need for revascularization in kidney transplant candidates [8]. Such a finding may have been due to the absence of a standard questionnaire, recall bias, accommodation to impaired performance, or a tendency to overstate health. A second study examined the utility of aerobic capacity, or peak oxygen intake $\left(\mathrm{pVO}_{2}\right)$, obtained through formal cardiopulmonary exercise testing (CPET) [11]. At a threshold of $17 \mathrm{~mL} / \mathrm{kg} / \mathrm{min}$, sensitivity of $\mathrm{pVO}_{2}$ in predicting cardiovascular events and all-cause mortality was $79-94 \%$ in kidney transplant candidates [11]. However, measuring $\mathrm{pVO}_{2}$ still requires specialized equipment and personnel, and abnormal results need to be "followed up" with formal CAD testing; the addition of CPET could therefore increase, rather than decrease, the overall test burden and health-care utilization of kidney transplant candidates.

The 6-minute walk test (6MWT) is a promising officebased tool that approximates $\mathrm{pVO}_{2}$ and could serve as a risk-stratification tool suitable for screening. The prog- nostic value of 6MWT has been demonstrated in numerous cardiopulmonary and vascular conditions, including heart failure, chronic obstructive pulmonary disease, pulmonary arterial hypertension, and peripheral arterial disease $[12,13]$. The $6 \mathrm{MWT}$ correlates directly with $\mathrm{pVO}_{2}$ and METs $(r=0.55-0.70)$ [12], including in patients with CKD [14]. Compared to self-report, the 6MWT is less prone to bias. Compared to $\mathrm{pVO}_{2}, 6 \mathrm{MWT}$ results are obtainable in routine clinical settings.

At our institution, we incorporated the 6MWT into the routine evaluation for medically complex deceased donor kidney transplant candidates nearing the top of our waitlist of 1,800-1,900 patients [15]. Organ shortage is acute in our area, and wait-time to a deceased donor kidney may exceed 10 years [16]. We therefore focused CAD assessment on patients who have survived to the top of waitlist. Our transplant team used 6MWT results to inform counselling on frailty, transplant candidacy, perioperative risk, and need for prehabilitation. Our team did not use 6MWT results to inform need for CAD testing or retesting, but adhered to our center-specific protocol outlined below.

We examined outcomes and CAD test results in our patients who underwent the 6MWT. Our main questions (and objectives) were as follows:

1. Does the 6MWT-based approach predict CAD-related events, and how does it perform compared to historyor risk factor-based approaches?

2. Does the 6MWT-based approach correlate with CAD test results, and how does it perform compared to history- or risk factor-based approaches?

3. How does the 6MWT-based approach perform compared to noninvasive $\mathrm{CAD}$ test results in predicting CAD-related events within 1 year of testing?

\section{Methods}

\section{Cohort Assembly}

We included all patients evaluated through our program's Transplant Readiness Assessment Clinic from May 2017, when we implemented the 6MWT, through April 2019, to allow at least 1 year of follow-up. The operational details of our deceased donor transplant candidate waitlist management strategy have been published previously $[15,16]$ (see online suppl. S1; for all online suppl. material, see www.karger.com/doi/10.1159/000516158 for details).

\section{CAD Testing}

CAD testing schedule adhered to our pre-established clinical algorithm (online suppl. S2). Specifically, our protocol required coronary angiography as the first-line test in patients with diabetic kidney disease and high-risk features [17], and revascularization of any flow-limiting stenoses was detected. 
Six-Minute Walk Testing

Experienced personnel from an affiliated Veterans Affairs Medical Center's Exercise Training Unit trained 3 transplant nurse coordinators to perform the $6 \mathrm{MWT}$, who conducted them at initial and follow-up visits. The training personnel performed withinsubject measurements periodically against the coordinators' measurements for quality assurance. Coordinators instructed patients to walk as fast as they could, at a safe pace, with their usual walking aid/prosthesis and/or with family assistance in the case of sensory impairment, in a quiet hallway between 2 marked objects delineating a distance of 100 feet over 6 min. Breaks and early stops were permitted and included in the $6 \mathrm{~min}$. Nonambulatory patients received a $6 \mathrm{MWT}$ result of 0 , and the reason for nonambulation was recorded. When a patient had multiple visits and multiple 6MWT results, we included the first result. Symptoms during testing were recorded; they were categorized as cardiac (chest pain, dyspnea, unexplained upper gastrointestinal symptoms with exertion, and fatigue [early stops due to lack of stamina]) or noncardiac (noncardiac pain and neuromuscular weakness). The tests were performed on the same day as the patient's clinic appointment and generally scheduled on nondialysis days, although we did not collect that information.

\section{Risk Stratification Approaches}

We analyzed 6MWT results in multiple ways: (1) as a continuous variable; (2) in a binary fashion using $400 \mathrm{~m}$ as a cut-off, based on prior studies demonstrating that $400 \mathrm{~m}$ corresponds to 4 METs [18-20]; and (3) designating 6MWT result $\geq 400 \mathrm{~m}$ and no cardiac symptoms provoked by $6 \mathrm{MWT}$ as low risk.

We defined self-reported cardiac symptoms as chest pain, dyspnea, or unexplained upper gastrointestinal symptom, elicited through history and review of systems. Of the risk factor guidelines, we chose the 2012 American Heart Association/American College of Cardiology Foundation Scientific Statement endorsed by the American Society of Transplantation (hereafter referred to as "AHA/ACCF criteria") [6], reported to be the most commonly used in a recent survey of kidney transplant professionals in the United States [4].

\section{Outcomes}

Our primary outcome was a CAD-related event, a composite of cardiac death, nonfatal myocardial infarction (defined as type 1 or 2 per the universal definition) [21], coronary revascularization (urgent and nonurgent), or removal from waitlist for advanced $\mathrm{CAD}$. We adjusted the analyses for competing events, including death from a noncardiac cause and waitlist removal for non-CADrelated reasons.

Our secondary outcome was CAD test results, performed within 6 months of the $6 \mathrm{MWT}$ and without intervening revascularization or a CAD-related event. We defined a positive myocardial perfusion scan as moderate or severe reversible ischemia, and a positive stress echocardiogram as a stress-induced wall motion abnormality. We defined a positive coronary angiogram as $\geq 70 \%$ stenosis or fractional flow reserve $\leq 0.80$ in a major epicardial vessel.

\section{Blinding}

In the choice of whether or not to perform CAD testing and which test to perform, we did not consider the 6MWT result, but rather adhered to our center protocol (online suppl. S2). The consulting cardiologists who performed and read the CAD tests were all blinded to the 6MWT results. A nephrologist (DJW) and a cardiologist (HA) performed independent adjudications of primary and secondary outcomes and resolved any discrepancy via a consensus format. Both were blinded to the exposure (6MWT result).

The transplant team was not blinded to the $6 \mathrm{MWT}$ result. The $6 \mathrm{MWT}$ formed a part of the patient's global physical function assessment (in conjunction with other tests including the sit-tostand tests and SF36 questionnaire) and used it to inform counselling on frailty, transplant candidacy, perioperative risk, and need for prehabilitation. However, we used no threshold of 6MWT to remove a patient from the waitlist or bar a patient from transplant. Further details are in our prior works [15].

\section{Analysis}

For objectives \#1 and \#3, wherein we examined the association between a 6MWT-based approach and CAD-related events, we applied the Fine and Grey subdistribution hazards models to examine time to a CAD-related event as the primary outcome, accounting for the competing events of noncardiac death and waitlist removal for non-CAD-related reasons. Using Kaplan-Meier product limit estimates, we built a time-dependent receiver operating characteristic curve $(\mathrm{ROC}(\mathrm{t}))$ and calculated the 1-year area-under-the-curve $(A U C(t))$ for the predictor in question. The ROC $(t)$ is a statistic generalizing the traditional ROC, developed for binary outcomes, to time-to-event analyses, by allowing the AUC to vary over time. We chose to compute the $\mathrm{AUC}(\mathrm{t})$ at 1 year, as most of our patients were within 1 year of a kidney transplant at the time of their 6MWT. We tested for interaction between the 6MWT and prespecific subgroups based on age and presence or absence of diabetes mellitus, $\mathrm{CAD}$, prior $\mathrm{CAD}$ testing, and noncardiac factors affecting the 6MWT (including lung disease, severe neuropathy or other neurological deficit, lower extremity amputations, active orthopedic issues, assistive walking device use, or noncardiac pain).

For objective \#2, we used logistic regression models to examine the association between the $6 \mathrm{MWT}$ and CAD test results. We tested for interaction between the 6MWT and the modality of CAD test (invasive vs. noninvasive).

\section{Companion Analysis}

In light of the high competing event rate, we performed a companion analysis in which we examined time to CAD-related event removing waitlist status change as a competing event in the subset of patients on whom we were able to obtain follow-up information. Here, we defined a CAD-related event as cardiac death, nonfatal myocardial infarction, or coronary revascularization. The only competing event was noncardiac death.

We used SAS Enterprise version 7.3 (Cary, NC, USA).

\section{Results}

\section{Baseline Characteristics}

Over the study period, we performed the 6MWT in 360 patients through TRAC (main cohort and cohort for objective \#1). To address the association between the 6MWT and CAD testing (objective \#2), we obtained 196 unique 6MWT-cardiac ischemia testing pairs from the 
Fig. 1. Cohort assembly. 6MWT, 6-minute walk test; TRAC, Transplant Readiness and Assessment Clinic.

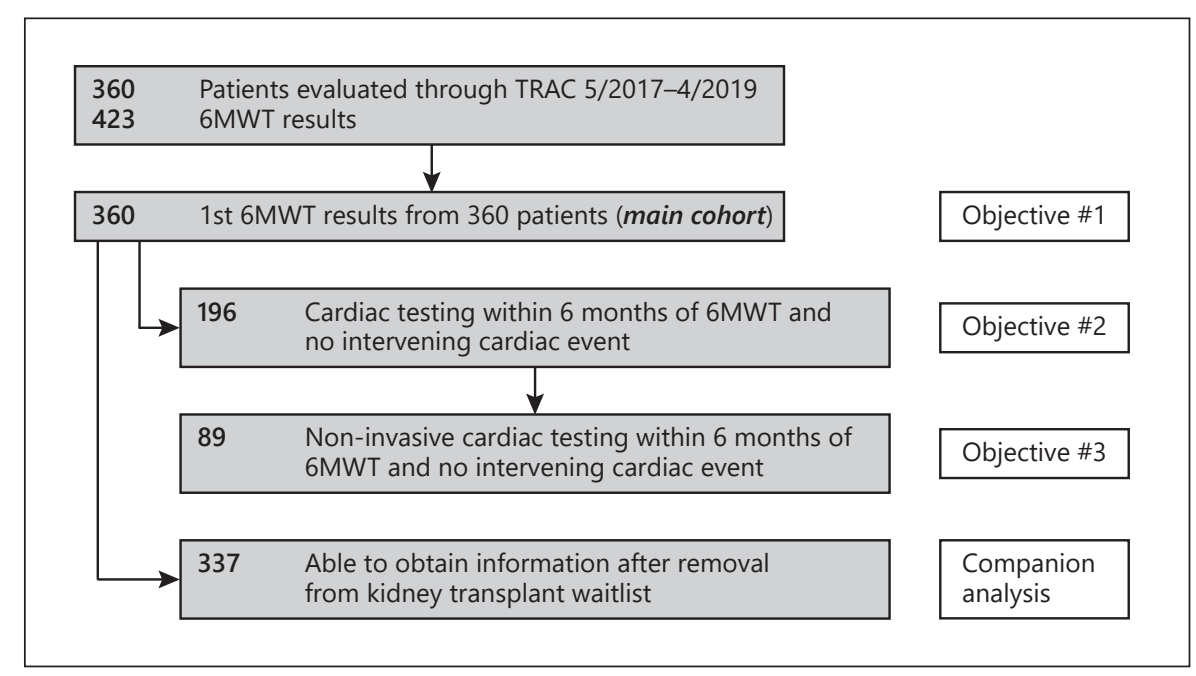

main cohort. To compare the 6MWT-based approach with noninvasive cardiac ischemia testing, we repeated the survival analysis in 89 patients who had noninvasive testing within 6 months of the $6 \mathrm{MWT}$ testing and without intervening $\mathrm{CAD}$-related event or revascularization (objective \#3). Figure 1 depicts details of cohort assembly.

Table 1 outlines the baseline characteristics of the main cohort. Patients with a $6 \mathrm{MWT}$ result $\geq 400 \mathrm{~m}$, compared to those with a $6 \mathrm{MWT}$ result $<400 \mathrm{~m}$, were younger, more likely to be male, less likely to have had a prior cardiac evaluation, and less likely to have atherosclerotic disease, diabetes mellitus, or noncardiac factors affecting the 6MWT. There was no appreciable difference in self-reported cardiac symptoms, but patients with $6 \mathrm{MWT}$ results $\geq 400 \mathrm{~m}$ were significantly less likely to have cardiac symptoms during the $6 \mathrm{MWT}$ or meet the AHA/ACCF criteria for cardiac screening. online suppl. S3 outlines the baseline characteristics of cohort \#2 and \#3 - the overall covariate distribution was similar to that of the main cohort, but due to the smaller sample sizes some of the differences were no longer statistically significant.

Patients with a 6 MWT result $\geq 400 \mathrm{~m}$ had a significantly longer follow-up time in our cohort, a lower likelihood of removal from the waitlist, and a higher likelihood of receiving a kidney transplant during follow-up, but the CAD test rate was comparable in both groups (Table 2).

Both a $6 \mathrm{MWT}$ result $\geq 400 \mathrm{~m}$ and a $6 \mathrm{MWT}$ result $\geq 400$ $\mathrm{m}$ plus no symptom during the $6 \mathrm{MWT}$ designated 45 and $44 \%$ of patients, respectively, as "low risk." In contrast, only $14 \%$ of patients met the AHA/ACCF criteria of "low risk." Only $19 \%$ patients had cardiac symptoms by his- tory, thus $81 \%$ patients were designated "low risk" by the self-reported symptom approach.

\section{Association with a CAD-Related Event}

Over a median follow-up of 499 (IQR 141-716) days, 50 (14\%) CAD-related events and $72(20 \%)$ competing events occurred in the main cohort $(N=360)$ (Table 2). The 6MWT-based approach was not significantly associated with CAD-related events within 1 year but was significantly associated with competing events (Table 3 ). Self-reported cardiac symptoms were not significantly associated with either CAD-related events or competing events. Not meeting AHA/ACCF criteria, in contrast, was significantly associated with a lower risk of CAD-related events within 1 year (sHR 0.11 [0.02-0.82]). Multivariate adjustment for age, sex, diabetes mellitus, any atherosclerotic disease, and presence of noncardiac factors affecting the 6MWT did not substantially change the results.

In our subgroup analysis, the association between 6MWT and CAD-related events was not significantly modified by age, sex or the presence or absence of diabetes, preexisting $\mathrm{CAD}$, prior CAD testing, or noncardiac factors affecting the 6MWT (Fig. 2). Furthermore, excluding the 17 patients who were nonambulatory did not alter the results noticeably (online suppl. S3).

\section{Association with Cardiac Ischemia Tests}

Of the 196 unique 6MWT-cardiac testing pairs, 102 (52\%) were invasive coronary angiography, while 94 (48\%) were noninvasive tests (52 stress echocardiogram, 38 myocardial perfusion scan, and 3 coronary computed tomography angiography). Forty-five (23\%) of the tests 
Table 1. Baseline characteristics of the main cohort, stratified by 6MWT results

\begin{tabular}{|c|c|c|c|}
\hline Baseline characteristic & $\begin{array}{l}6 \mathrm{MWT}<400 \mathrm{~m} \\
(N=199)\end{array}$ & $\begin{array}{l}6 \mathrm{MWT} \geq 400 \mathrm{~m} \\
(N=161)\end{array}$ & $p$ value \\
\hline \multicolumn{4}{|l|}{ Demographics } \\
\hline Age, years & $58 \pm 10$ & $50 \pm 12$ & $<0.0001$ \\
\hline Sex (\% male) & $90(45)$ & $106(66)$ & $<0.0001$ \\
\hline \multicolumn{4}{|l|}{ Race, $n(\%)$} \\
\hline White & $34(17)$ & $18(11)$ & \multirow{5}{*}{0.5} \\
\hline Black & $18(9)$ & $14(9)$ & \\
\hline Hispanic & $91(46)$ & $81(50)$ & \\
\hline Asian & $45(23)$ & $41(25)$ & \\
\hline Other & $11(6)$ & $7(4)$ & \\
\hline Time from listing, years & $6.1 \pm 3.1$ & $5.8 \pm 2.9$ & 0.3 \\
\hline \multicolumn{4}{|l|}{ Cardiac risk factors and comorbidities, $n(\%)$} \\
\hline CAD testing prior to visit & $142(71)$ & $99(61)$ & 0.05 \\
\hline CAD with revascularization & $43(22)$ & $26(16)$ & 0.2 \\
\hline Any atherosclerotic disease (CAD equivalent $)^{1}$ & $86(43)$ & $36(22)$ & $<0.0001$ \\
\hline Dialysis vintage, years & $6.9 \pm 3.3$ & $6.6 \pm 3.3$ & 0.3 \\
\hline \multicolumn{4}{|l|}{ Dialysis modality, $n(\%)$} \\
\hline Hemodialysis & $159(82)$ & $128(84)$ & \multirow{2}{*}{0.9} \\
\hline Peritoneal dialysis & $34(18)$ & $25(16)$ & \\
\hline Diabetes mellitus & $121(61)$ & $52(33)$ & $<0.0001$ \\
\hline Hypertension & $181(91)$ & $146(91)$ & 0.9 \\
\hline Any history of smoking & $57(29)$ & $68(42)$ & 0.007 \\
\hline Noncardiac factors affecting the $6 \mathrm{MWT}$ result & $107(54)$ & $30(19)$ & $<0.0001$ \\
\hline Lung disease $^{2}$ & $26(13)$ & $18(11)$ & 0.6 \\
\hline Neurologic weakness & $5(3)$ & $0(0)$ & $0.07^{*}$ \\
\hline Lower extremity amputation & $24(12)$ & $4(2)$ & 0.003 \\
\hline Active orthopedic issue ${ }^{3}$ & $26(13)$ & $1(1)$ & $<0.0001$ \\
\hline Assistive walking device use $\mathrm{e}^{4}$ & $57(29)$ & $1(1)$ & $<0.0001$ \\
\hline Noncardiac pain & $29(16)$ & $8(5)$ & $<0.0001$ \\
\hline \multicolumn{4}{|l|}{ Cardiac symptoms, screening, and testing } \\
\hline Cardiac symptoms $s^{5}$ at rest & $22(11)$ & $12(7)$ & 0.2 \\
\hline Cardiac symptoms ${ }^{5}$ during $6 \mathrm{MWT}$ & $42(21)$ & $2(1)$ & $<0.0001$ \\
\hline Meets AHA/ACCF criteria ${ }^{6}$ for screening & $181(91)$ & $126(78)$ & 0.0007 \\
\hline CAD testing within 6 months & $83(42)$ & $70(43)$ & 0.7 \\
\hline
\end{tabular}

6MWT, 6-minute walk test; CAD, coronary artery disease. * Calculated by Fisher's exact test due to small cell size. ${ }^{1}$ Any atherosclerotic disease (CAD equivalent): any history of revascularized CAD, ischemic cerebrovascular accidents, or peripheral artery disease (active claudication, revascularization, or amputation). ${ }^{2}$ Lung disease: airway disease (chronic obstructive pulmonary disease, reactive airway disease, or adult asthma) or parenchymal lung disease. ${ }^{3}$ Active orthopedic issue or nonweight-bearing: issues impairing locomotion, including fracture, casts/prosthesis/boots, or active lower extremity ulcer requiring nonweight-bearing. ${ }^{4}$ Assistive devise use: cane or walker use. ${ }^{5}$ Cardiac symptoms: chest pain, dyspnea on exertion, unexplained upper gastrointestinal symptoms with exertion, or fatigue requiring stopping during the 6MWT. ${ }^{6} \mathrm{AHA} / \mathrm{ACCF}$ criteria: from the 2012 American Heart Association/American College of Cardiology Foundation Scientific Statement endorsed by the American Society of Transplantation, ${ }^{3}$ high risk is defined as having CAD or CAD-equivalent, diabetes mellitus, or 2 or more risk factors including hypertension, age ( $>45$ for men or $>55$ for women), cigarette smoking, or left ventricular hypertrophy. Dyslipidemia and family history were 2 additional risk factors that we did not have in our database.

were deemed "positive," including 34 (33\%) coronary angiograms and $11(11 \%)$ positive noninvasive tests. The 6MWT-based approach did not predict positive CAD test results (Table 4, AUC 0.54-0.55), neither did self-report- ed cardiac symptoms or AHA/ACCF criteria (Table 4, AUC 0.51-0.52). The results did not differ based on the modality of cardiac ischemia testing (noninvasive vs. invasive, $p$ for interaction $>0.05$, online suppl. S4). 
Table 2. Outcomes of the main cohort, stratified by $6 \mathrm{MWT}$ results

\begin{tabular}{|c|c|c|c|}
\hline Outcome & $\begin{array}{l}6 \mathrm{MWT}<400 \mathrm{~m} \\
(N=199)\end{array}$ & $\begin{array}{l}6 \mathrm{MWT} \geq 400 \mathrm{~m} \\
(N=161)\end{array}$ & $p$ value \\
\hline Follow-up time, days & $389 \pm 337$ & $545 \pm 276$ & $<0.0001$ \\
\hline Received a kidney transplant during follow-up, $n(\%)$ & $51(26)$ & $85(53)$ & $<0.0001$ \\
\hline \multicolumn{4}{|l|}{ Primary outcome: CAD-related event, $n(\%)$} \\
\hline Cardiac death & $6(3)$ & $3(2)$ & \multirow{8}{*}{$<0.0001$} \\
\hline Nonfatal MI & $9(5)$ & $4(2)$ & \\
\hline Type 1 & 6 & 3 & \\
\hline Type 2 & 3 & 1 & \\
\hline Revascularization & $14(7)$ & $10(6)$ & \\
\hline Urgent & 2 & 1 & \\
\hline Nonurgent & 12 & 9 & \\
\hline Removal from waitlist for advanced CAD & $3(2)$ & $1(1 \%)$ & \\
\hline Removed from waitlist & $64(32)$ & $5(3)$ & $<0.0001$ \\
\hline Follow-up available after waitlist removal & $46(72)$ & $5(100)$ & 0.2 \\
\hline Noncardiac death on the waitlist & $5(3)$ & 2 & \\
\hline CAD testing within 6 months & $83(42)$ & $70(43)$ & 0.7 \\
\hline
\end{tabular}

CAD, Coronary artery disease; MI, myocardial infarction; 6MWT, 6-min walk test.

Table 3. Identification of a low-risk cohort: prediction of CAD-related event within 1 year in a cohort of 360 patients

\begin{tabular}{|c|c|c|c|c|}
\hline Predictor of low risk & $\begin{array}{l}N(\%) \text { meeting } \\
\text { low-risk definition }\end{array}$ & $\begin{array}{l}\text { sHR for } \\
\text { CAD-related event }\end{array}$ & $\begin{array}{l}\text { sHR for } \\
\text { competing event }\end{array}$ & $\operatorname{AUC}(\mathrm{t})$ \\
\hline $6 \mathrm{MWT}$ result (continuous, per $50 \mathrm{~m}$ ) & $\mathrm{n} / \mathrm{a}$ & $1.00(0.90-1.11)$ & $0.70(0.66-0.75)$ & 0.55 \\
\hline $6 \mathrm{MWT}$ result $\geq 400 \mathrm{~m}+$ no cardiac symptom during $6 \mathrm{MWT}$ & $159(44)$ & $0.70(0.39-1.24)$ & $0.08(0.03-0.20)$ & 0.55 \\
\hline No self-reported cardiac symptom & $326(81)$ & $0.89(0.36-2.21)$ & $0.77(0.37-1.57)$ & 0.50 \\
\hline Does not meet AHA/ACCF criteria* & $53(14)$ & $0.11(0.02-0.82)$ & $0.50(0.22-1.11)$ & 0.54 \\
\hline
\end{tabular}

Statistically significant results are bolded. AUC(t) refers to the area-under-the-curve for 1-year time-dependent receiver operator curve for the CAD-related event. 6MWT, 6-min walk test; sHR, subproportional hazards ratio; CAD, coronary artery disease. ${ }^{*}$ AHA/ ACCF criteria: from the 2012 American Heart Association/American College of Cardiology Foundation Scientific Statement endorsed by the American Society of Transplantation, ${ }^{3}$ high risk is defined as having CAD or CAD-equivalent, diabetes mellitus, or 2 or more risk factors including hypertension, age ( $>45$ for men or $>55$ for women), cigarette smoking, or left ventricular hypertrophy. Dyslipidemia and family history were 2 additional risk factors that we did not have in our database.

\section{Comparison of the 6MWT-Based Approach with}

Noninvasive Cardiac Ischemia Tests

Among a subcohort of 89 patients with a $6 \mathrm{MWT}$ and a noninvasive CAD test within 6 months of the $6 \mathrm{MWT}, 8$ (9\%) CAD-related events and 12 (13\%) competing events occurred over a median follow-up of 577 (IQR 424-750) days. Of the $8 \mathrm{CAD}$-related events, 2 were type- $1 \mathrm{MI}, 2$ were waitlist removal for CAD, 1 was cardiac death, 2 were nonurgent revascularization, and 1 was urgent revascularization. Of the 12 competing events, 8 were waitlist removal for non-CAD reasons and 4 were noncardiac death. Similar to the main analysis, the 6MWT-based approach was not significantly associated with CAD-related events within 1 year but was significantly associated with competing events (Table 5). A negative CAD test was significantly associated with a lower risk of CAD-related events within 1 year (sHR 0.08 [0.02-0.33]).

\section{Association with CAD-Related Events within 1 Year,} Regardless of Kidney Transplant Waitlist Status

In the companion analysis, we extended follow-up for CAD-related events beyond waitlist removal, where pos- 
Fig. 2. Subgroup analysis: associations between 6MWT and CAD-related event by sHR. All $p$ values for testing by interaction were $>0.05$, suggesting no detectable effect modification. 6MWT, 6-minute walk test; sHR, subproportional hazard ratio; CAD, coronary artery disease.

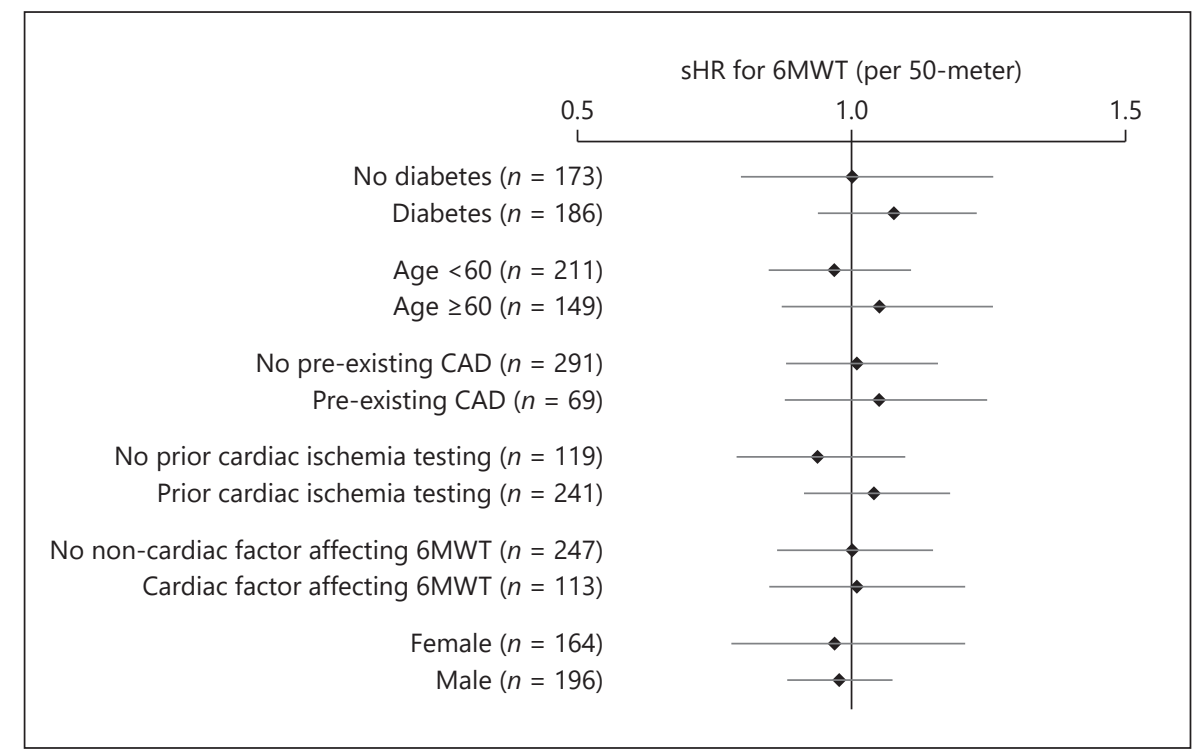

Table 4. Concordance between risk stratification and CAD testing in a cohort of 196 patients

\begin{tabular}{|c|c|c|c|}
\hline Predictor & $\begin{array}{l}N(\%) \text { meeting } \\
\text { low-risk definition }\end{array}$ & OR & AUC \\
\hline $6 \mathrm{MWT}$ result (continuous, per $50 \mathrm{~m}$ ) & $\mathrm{n} / \mathrm{a}$ & $0.96(0.81-1.13)$ & 0.54 \\
\hline $6 \mathrm{MWT}$ result $\geq 400 \mathrm{~m}$ & $89(45)$ & $0.68(0.35-1.35)$ & 0.55 \\
\hline $6 \mathrm{MWT}$ result $\geq 400 \mathrm{~m}+$ no cardiac symptom during $6 \mathrm{MWT}$ & $88(45)$ & $0.70(0.35-1.39)$ & 0.54 \\
\hline No cardiac symptom at rest & $185(94)$ & $1.67(0.36-7.81)$ & 0.51 \\
\hline Does not meet AHA/ACCF criteria* & $20(10)$ & $0.57(0.16-2.05)$ & 0.52 \\
\hline
\end{tabular}

None of the results were statistically significant, i.e. all of the $95 \%$ confidence intervals for odds ratio crossed 1.0. 6MWT, 6-minute walk test; OR, odds ratio; AUC, area under the curve; CAD, coronary artery disease. * AHA/ACCF criteria: from the 2012 American Heart Association/American College of Cardiology Foundation Scientific Statement endorsed by the American Society of Transplantation, high risk is defined as having CAD or CAD-equivalent, diabetes mellitus, or 2 or more risk factors including hypertension, age ( $>45$ for men or $>55$ for women), cigarette smoking, or left ventricular hypertrophy. Dyslipidemia and family history were 2 additional risk factors that we did not have in our database.

sible. Of the 69 patients who were removed from the waitlist, we were unable to obtain any clinical information after waitlist removal in 23 and excluded them, leaving a sub-cohort of 337 patients (Fig. 1). Over a median followup of 575 (IQR 391-750) days, 53 CAD-related events and 12 competing events (noncardiac deaths) occurred. Of the 53 CAD-related events, 9 were type- 1 MI, 5 were type-2 MI, 13 were cardiac death, 21 were nonurgent revascularization, and 5 were urgent revascularization. A higher 6MWT result was significantly associated with a lower risk of CAD-related event (sHR 0.92 [0.84-1.00] per every $50 \mathrm{~m}$, AUC 0.56 , Table 6 ). A low-risk category, defined as $6 \mathrm{MWT} \geq 400 \mathrm{~m}$ or $6 \mathrm{MWT} \geq 400$ plus no symp- toms during 6MWT, was associated with approximately $50 \%$ lower risk of CAD-related event, but AUC remained low (Table 6).

\section{Discussion}

In this study, we investigated using the 6MWT to riskstratify patients with high priority on the kidney transplant waitlist to inform novel screening strategies for ischemic heart disease in this challenging patient population. The patients' treatment team and the research staff were blinded to the $6 \mathrm{MWT}$ results while deciding on di- 
Table 5. Identification of a low-risk cohort: prediction of CAD-related event within 1 year in a cohort of 89 patients with noninvasive cardiac ischemia testing

\begin{tabular}{|c|c|c|c|c|}
\hline Predictor of low risk & $\begin{array}{l}N(\%) \text { meeting } \\
\text { low-risk definition }\end{array}$ & $\begin{array}{l}\text { sHR for } \\
\text { CAD-related event }\end{array}$ & $\begin{array}{l}\text { sHR for } \\
\text { competing event }\end{array}$ & $\operatorname{AUC}(\mathrm{t})$ \\
\hline $6 \mathrm{MWT}$ result (continuous, per $50 \mathrm{~m}$ ) & $\mathrm{n} / \mathrm{a}$ & $1.14(0.90-1.46)$ & $0.61(0.49-0.78)$ & 0.58 \\
\hline $6 \mathrm{MWT}$ result $\geq 400 \mathrm{~m}+$ no cardiac symptom during $6 \mathrm{MWT}$ & $50(56)$ & $0.81(0.20-3.27)$ & $0.25(0.07-0.91)$ & 0.46 \\
\hline Negative CAD test result & $79(89)$ & $0.08(0.02-0.33)$ & $0.57(0.13-2.48)$ & 0.70 \\
\hline
\end{tabular}

Statistically significant results are bolded. AUC(t) refers to the area-under-the-curve for 1-year time-dependent receiver operator curve for the CAD-related event. sHR, subproportional hazards ratio; CAD, coronary artery disease; 6MWT, 6-minute walk test.

Table 6. Companion analysis accounting for CAD-related event after waitlist removal: prediction of CAD-related event in a cohort of 337 patients

\begin{tabular}{|c|c|c|c|c|}
\hline Predictor of low risk & $\begin{array}{l}N(\%) \text { meeting } \\
\text { low-risk definition }\end{array}$ & $\begin{array}{l}\text { sHR for } \\
\text { CAD-related event }\end{array}$ & $\begin{array}{l}\text { sHR for } \\
\text { competing event }\end{array}$ & $\mathrm{AUC}(\mathrm{t})$ \\
\hline $6 \mathrm{MWT}$ result (continuous, per $50 \mathrm{~m}$ ) & $\mathrm{n} / \mathrm{a}$ & $0.92(0.84-1.00)$ & $0.91(0.78-1.06)$ & 0.56 \\
\hline $6 \mathrm{MWT}$ result $\geq 400 \mathrm{~m}+$ no cardiac symptom during $6 \mathrm{MWT}$ & $157(47)$ & $0.52(0.30-0.93)$ & $0.39(0.11-1.45)$ & 0.56 \\
\hline No self-reported cardiac symptom & $306(91)$ & $0.88(0.35-2.23)$ & $0.45(0.10-2.05)$ & 0.51 \\
\hline Does not meet AHA/ACCF criteria* & $53(16)$ & $0.10(0.01-0.75)$ & $1.18(0.26-5.40)$ & 0.53 \\
\hline
\end{tabular}

Statistically significant results are bolded. AUC(t) refers to the area-under-the-curve for 1-year time-dependent receiver operator curve for the CAD-related event. 6MWT, 6-minute walk test; sHR, subproportional hazards ratio; AUC(t), area-under-the-curve for 1 -year time-dependent receiver operator curve; CAD, coronary artery disease. * AHA/ACCF criteria: from the 2012 American Heart Association/American College of Cardiology Foundation Scientific Statement endorsed by the American Society of Transplantation, high risk is defined as having CAD or CAD-equivalent, diabetes mellitus, or 2 or more risk factors including hypertension, age ( $>45$ for men or $>55$ for women), cigarette smoking, or left ventricular hypertrophy. Dyslipidemia and family history were 2 additional risk factors that we did not have in our database.

agnostic testing, waitlist removal, or adjudicating study outcomes. Against a high background of competing events, especially waitlist removal for non-CAD reasons, we did not see an association between the 6MWT and CAD-related events. When we omitted kidney transplant waitlist status in the sensitivity analysis, focusing only on CAD-related events and the competing event of noncardiac death, we saw a modest association between the 6MWT and CAD-related events.

The null finding in our primary analysis is surprising, based on preexisting literature on the 6MWT and a prior study demonstrating the strong association between cardiac events and $\mathrm{pVO}_{2}$, of which the $6 \mathrm{MWT}$ is a surrogate, in kidney transplant candidates [11]. As the relation between the $6 \mathrm{MWT}$ and $\mathrm{pVO}_{2}$ is linear over $10-20 \mathrm{~mL} / \mathrm{min} /$ $\mathrm{kg}$ [22], a range which encompasses Chakkera et al.'s [11] $\mathrm{pVO}_{2}$ threshold for pretransplant CAD screening, we hypothesized that the 6MWT would perform as well as
$\mathrm{pVO}_{2}$. A few explanations are possible. In the $\mathrm{pVO}_{2}$ study, the $\mathrm{CAD}$ screening protocol incorporated $\mathrm{pVO}_{2}$; in other words, patients who had a good $\mathrm{pVO}_{2}$ did not undergo additional CAD testing. Such a study design necessarily overestimated the sensitivity and specificity of the index test. Our study was performed blinded to the 6MWT result and we were much more likely to recommend upfront coronary angiography in patients with diabetes mellitus (over 50\% in our cohort). We therefore detected, and intervened on, asymptomatic disease at a higher rate. Another explanation of the null finding is the high event rate for competing event and the strong negative association between the $6 \mathrm{MWT}$ and the competing event. The $6 \mathrm{MWT}$, or physical performance, is a snapshot of the patient's overall physical function and health state, of which cardiorespiratory fitness is a part. In this study, as in our previous study [15], the 6MWT was strongly associated with waitlist removal and death. Such an association bi- 
ased the association between the 6MWT and CAD-related events toward the null. Patients with a high 6MWT result were more likely to remain on our transplant waitlist, and any CAD-related events they developed would be captured as an endpoint. In contrast, patients with a low $6 \mathrm{MWT}$ result had a $32 \%$ probability of waitlist removal (Table 2); any CAD-related event they developed after waitlist removal would not be captured. Indeed, the positive results of our companion analysis appear to support the explanation that the higher waitlist removal rate in patients with low 6MWTs results may have confounded the association between 6MWT and CAD-related events.

Based on this observation, we contend that any consideration of cardiac risk stratification in the prevalent kidney transplant candidate population must consider the high background of waitlist removal and death for noncardiac reasons. Advances and successes in cardiology, especially in the medical management of CAD, have translated into a declining significance of CAD as a cause of death in both the pretransplant $[23,24]$ and posttransplant phases of ESKD [25]. In our study, while the 6MWT appears to be associated with CAD-related events in our companion analysis, the effect is overshadowed in the primary analysis by other medical factors and processes. An implication is that any future CAD screening strategy ought to only be applied among patients who have a reasonable chance of surviving and receiving a kidney transplant in the near future. The role of the 6MWT, given its strong association with all-cause mortality on the kidney transplant waitlist [15], may be then to identify such patients, rather than simply to risk-stratify for CAD. Indeed, the ongoing organ shortage means that the probability of dying on or being removed from the kidney waitlist may exceed the probability of receiving a transplant in many parts of the country, particularly for elderly patients [26, 27]. Our findings thus have far ranging implications for the area of CAD screening and waitlist management for kidney transplant candidates.

How does the 6MWT-based approach compare to other approaches of risk stratification? Self-reported symptoms designated the most (81\%) patients at low risk, but was no more predictive of CAD-related events than chance (AUC 0.50). The AHA/ACCF criteria, in contrast, only designated $14 \%$ of patients as low risk and not needing testing. Meeting the AHA/ACCF criteria was significantly associated with CAD-related events; we suspect that this is at least partly because our CAD testing criteria overlaps largely with the AHA/ACCF criteria, especially as all patients with diabetic kidney disease (also one of the AHA/ACCF risk factors) underwent coronary angiogra- phy as a first-line test in our cohort. Negative CAD testing was associated with a lower rate of CAD events with an AUC of 0.70 , suggesting moderate discriminatory ability along the lines of what has been reported (reviewed in Hart et al. [1]). However, such an association is clearly confounded, as patients with negative CAD test results were unlikely to be followed up with more invasive testing. Finally, none of the risk stratification approaches, whether the 6MWT-, symptom-, or risk factor-based, correlated well with CAD test results. Overall, all the risk stratification approaches we evaluated in this study have their shortcomings, as described above.

Our study does not address whether the high elective revascularization rate in our patient cohort is appropriate. Our center's protocol was based on the a small, singlecenter trial in Lancet from the 1990s, which showed a 5 -fold reduction in cardiac events from coronary revascularization over medical treatment in kidney transplant candidates with type 1 diabetes mellitus [28], which our center subsequently validated [17]. Medical treatment in that study consisted of only aspirin and calcium channel blocker, and its extraordinarily high event rate has not been replicated elsewhere. In a recent web-based survey of US kidney transplant programs, a full $26 \%$ reported using the coronary angiogram as first-line in dialysis-dependent kidney transplant candidates [4]. Our center practice, although aggressive, therefore accorded with a sizable minority of US transplant programs. In the recently completed, multicenter ISCHEMIA-CKD trial, where contemporary standards of medical management were applied, there was no obvious advantage to revascularization in patients with stable CAD and CKD, including those with ESKD [2]. What is the benefit of revascularization in patients with obstructive CAD on coronary angiography, but no symptoms and, importantly, good physical performance as evidenced by good 6MWT performance? This is an open question that we hope to address with future studies.

This study has several strengths. We assembled a sample diverse in terms of age, sex, race, ethnicity, and duration of ESKD. We conducted analyses accounting for competing risks, methods essential in populations with multiple comorbidities. There are also multiple weaknesses. Because these patients represent those at the "top of the waitlist," and because our regions has one of the longest kidney waitlists in the country, these results may not generalize to all kidney transplant candidates. Reflecting this long waitlist and the fact that our cohort is created from a "top-of-the-waitlist" clinic, our rate of waitlist removal was quite high over the study period. 
Whether 6MWT-based risk stratification will be similarly limited if applied early in the transplant evaluation process, before the patients have accrued comorbidities that place them at risk for waitlist removal and complicate the interpretation of 6MWT, is an open question. Our center's protocol favors upfront use of invasive coronary angiography rather than noninvasive testing, in contrast with most other kidney transplant programs [4], and hence our rate of revascularization in asymptomatic patients will likely be higher than at other centers, inflating our CAD-related event rate.

In conclusion, we find that all modes of risk stratification we examined, be they performance-, symptom-, or risk factor-based, are limited in a cohort of prevalent waitlisted kidney transplant candidates with high comorbidity burden and likelihood of CAD event. The 6MWT designates fewer patients as high risk and in need of further testing (compared to risk factor-based approaches), but its utility as a pure CAD risk stratification tool is modulated by the background waitlist removal rate.

\section{Acknowledgement}

We acknowledge Ms. Lynn Clinton for her assistance in database management.

\section{Statement of Ethics}

The Stanford Institutional Review Board approved this project (protocol \#43639) which we conducted in adherence with the Declaration of Helsinki, and waived the need for individual informed consent.

\section{Conflict of Interest Statement}

The authors have no conflicts of interest to disclose.

\section{Funding Sources}

This work supported by funding from the American Heart Association under grant 19CDA34490021 (X.C.), National Institute of Diabetes and Digestive and Kidney Diseases under grant K23 DK123410-1 (X.C.), and the Sobrato Gift Fund (J.T.).

\section{Author Contributions}

Conception and design: X.C., G.C., J.T., and W.F. Data acquisition, analysis, and interpretation: X.C., D.W., H.A., and M.S. Drafting and critical revision: X.C., M.S., G.C., J.T., and W.F. Final approval: all authors. Agreement to be accountable: X.C., J.T., and W.F.

\section{References}

1 Hart A, Weir MR, Kasiske BL. Cardiovascular risk assessment in kidney transplantation. Kidney Int. 2015;87(3):527-34.

2 Bangalore S, Maron DJ, O'Brien SM, Fleg JL, Kretov EI, Briguori C, et al. Management of coronary disease in patients with advanced kidney disease. N Engl J Med. 2020;382(17): 1608-18.

3 Nimmo A, Forsyth JL, Oniscu GC, Robb M, Watson C, Fotheringham J, et al. A propensity score matched analysis indicates screening for asymptomatic coronary artery disease does not predict cardiac events in kidney transplant recipients. Kidney Int. 2021;99(2): 431-42.

4 Cheng XS, Mathew RO, Parasuraman R, Tantisattamo E, Levea SL, Kapoor R, et al. Coronary artery disease screening of asymptomatic kidney transplant candidates: a webbased survey of practice patterns in the United States. Kidney Med. 2020 Jul-Aug;2(4):5057.

5 Herzog CA, Asinger RW, Berger AK, Charytan DM, Díez J, Hart RG, et al. Cardiovascular disease in chronic kidney disease. A clinical update from kidney disease: improving global outcomes (KDIGO). Kidney Int. 2011;80(6): $572-86$.
6 Lentine KL, Costa SP, Weir MR, Robb JF, Fleisher LA, Kasiske BL, et al. Cardiac disease evaluation and management among kidney and liver transplantation candidates: a scientific statement from the American Heart Association and the American College of Cardiology Foundation. J Am Coll Cardiol. 2012; 60(5):434-80

7 Israni AK, Snyder JJ, Skeans MA, Peng Y, Maclean JR, Weinhandl ED, et al. Predicting coronary heart disease after kidney transplantation: Patient Outcomes in Renal Transplantation (PORT) Study. Am J Transplant. 2010; 10(2):338-53

8 Friedman SE, Palac RT, Zlotnick DM, Chobanian MC, Costa SP. A call to action: variability in guidelines for cardiac evaluation before renal transplantation. Clin J Am Soc Nephrol. 2011;6(5):1185-91.

9 Lentine KL, Schnitzler MA, Brennan DC, Snyder JJ, Hauptman PJ, Abbott KC, et al. Cardiac evaluation before kidney transplantation: a practice patterns analysis in medicare-insured dialysis patients. Clin J Am Soc Nephrol. 2008;3(4):1115-24.

10 Fleisher LA, Fleischmann KE, Auerbach AD, Barnason SA, Beckman JA, Bozkurt B, et al. 2014 ACC/AHA guideline on perioperative cardiovascular evaluation and management of patients undergoing noncardiac surgery: a report of the American College of Cardiology/American Heart Association Task Force on practice guidelines. J Am Coll Cardiol. 2014;64(22): e77-137.

11 Chakkera HA, Angadi SS, Heilman RL, Kaplan B, Scott RL, Bollempalli H, et al. Cardiorespiratory fitness (peak oxygen uptake): safe and effective measure for cardiovascular screening before kidney transplant. J Am Heart Assoc. 2018;7(11):e008662.

12 Rasekaba T, Lee AL, Naughton MT, Williams TJ, Holland AE. The six-minute walk test: a useful metric for the cardiopulmonary patient. Intern Med J. 2009;39(8):495-501.

13 Montgomery PS, Gardner AW. The clinical utility of a six-minute walk test in peripheral arterial occlusive disease patients. J Am Geriatr Soc. 1998;46(6):706-11.

14 de Moraes Kohl L, Signori LU, Ribeiro RA, Silva AM, Moreira PR, Dipp T, et al. Prognostic value of the six-minute walk test in endstage renal disease life expectancy: a prospective cohort study. Clinics. 2012;67(6):581-6. 
15 Cheng XS, Myers JN, Han J, Stedman MR, Watford DJ, Lee J, et al. Physical performance testing in kidney transplant candidates at the top of the waitlist. Am J Kidney Dis. 2020; 76(6):815-25.

16 Cheng XS, Busque S, Lee J, Discipulo K, Hartley C, Tulu Z, et al. A new approach to kidney waitlist management in the kidney allocation system era: pilot implementation and evaluation. Clin Transplant. 2018;32(11):e13406.

17 Ma IW, Valantine HA, Shibata A, Waskerwitz J, Dafoe DC, Alfrey EJ, et al. Validation of a screening protocol for identifying low-risk candidates with type 1 diabetes mellitus for kidney with or without pancreas transplantation. Clin Transplant. 2006;20(2):139-46.

18 Ross RM, Murthy JN, Wollak ID, Jackson AS. The six minute walk test accurately estimates mean peak oxygen uptake. BMC Pulm Med. 2010;10:31.

19 Balady GJ, Arena R, Sietsema K, Myers J, Coke L, Fletcher GF, et al. Clinician's guide to cardiopulmonary exercise testing in adults: a scientific statement from the American Heart Association. Circulation. 2010;122(2):191225.

20 Gomberg-Maitland M, Huo D, Benza RL, McLaughlin VV, Tapson VF, Barst RJ. Creation of a model comparing 6-minute walk test to metabolic equivalent in evaluating treatment effects in pulmonary arterial hypertension. J Heart Lung Transplant. 2007;26(7): 732-8.

21 Thygesen K, Alpert JS, Jaffe AS, Chaitman BR, Bax JJ, Morrow DA, et al. Fourth universal definition of myocardial infarction (2018). Circulation. 2018;138(20):e618-51.

22 Poole-Wilson PA. The 6-minute walk. A simple test with clinical application. Eur Heart J. 2000;21(7):507-8.

23 Wakasugi M, Kazama JJ, Narita I. Mortality trends among Japanese dialysis patients, 1988-2013: a joinpoint regression analysis. Nephrol Dial Transplant. 2016;31(9):1501-7.

24 Storey BC, Staplin N, Harper CH, Haynes R, Winearls CG, Goldacre R, et al. Declining co- morbidity-adjusted mortality rates in English patients receiving maintenance renal replacement therapy. Kidney Int. 2018;93(5):1165-74.

25 Ying T, Shi B, Kelly PJ, Pilmore H, Clayton PA, Chadban SJ. Death after kidney transplantation: an analysis by era and time posttransplant. J Am Soc Nephrol. 2020;31(12): 2887-99.

26 Hart A, Smith JM, Skeans MA, Gustafson SK, Wilk AR, Castro S, et al. OPTN/SRTR 2018 annual data report: kidney. Am J Transplant. 2020;20(Suppl s1):20-130.

27 Schold J, Srinivas TR, Sehgal AR, Meier-Kriesche HU. Half of kidney transplant candidates who are older than 60 years now placed on the waiting list will die before receiving a deceased-donor transplant. Clin J Am Soc Nephrol. 2009;4(7):1239-45.

28 Manske CL, Wang Y, Rector T, Wilson RF, White CW. Coronary revascularisation in insulin-dependent diabetic patients with chronic renal failure. Lancet. 1992;340(8826):9981002 . 